\title{
Optimizing purebred selection for crossbred performance using QTL with different degrees of dominance
}

\author{
Jack C.M. DekKers*', Reena ChaKraborty \\ Department of Animal Science, 225C Kildee Hall, Iowa State University, Ames, \\ IA, 50011, USA
}

(Received 9 April 2003; accepted 17 December 2003)

\begin{abstract}
A method was developed to optimize simultaneous selection for a quantitative trait with a known QTL within a male and a female line to maximize crossbred performance from a two-way cross. Strategies to maximize cumulative discounted response in crossbred performance over ten generations were derived by optimizing weights in an index of a QTL and phenotype. Strategies were compared to selection on purebred phenotype. Extra responses were limited for QTL with additive and partial dominance effects, but substantial for QTL with over-dominance, for which optimal QTL selection resulted in differential selection in male and female lines to increase the frequency of heterozygotes and polygenic responses. For overdominant QTL, maximization of crossbred performance one generation at a time resulted in similar responses as optimization across all generations and simultaneous optimal selection in a male and female line resulted in greater response than optimal selection within a single line without crossbreeding. Results show that strategic use of information on over-dominant QTL can enhance crossbred performance without crossbred testing.
\end{abstract}

crossbreeding / selection / quantitative trait loci / marker assisted selection

\section{INTRODUCTION}

In most livestock production systems, crossbreds are used for commercial production to capitalize on heterosis and complementarity and the aim of selection within pure-lines is to maximize crossbred performance. Selection is, however, within pure-lines and primarily based on purebred data, which may not maximize genetic progress in crossbred performance [21]. Several theoretical studies have shown that selection on a combination of crossbred and purebred performance can result in greater responses in crossbred performance, in particular if genes with complete or over-dominance affect the trait [1,20,22].

*Corresponding author: jdekkers@iastate.edu 
Collection of crossbred data, however, requires separate testing and recording strategies.

Molecular genetics has enabled the identification of quantitative trait loci (QTL) for many traits of interest in livestock. The strategic use of non-additive QTL in pure-line selection allows selection for crossbred performance without crossbred data. For non-additive QTL, Dekkers [4] showed that the breeding value of the QTL that maximizes the genetic level of progeny depends on the frequency of the QTL among mates and that extra gains of up to $9 \%$ could be obtained over a single generation for overdominant QTL at intermediate frequency by optimizing QTL breeding values. In practice, however, the goal is to maximize gains in current and future generations. Several studies have shown that with selection on QTL, maximization of response in the short term can result in lower cumulative responses in the longer term [10, 12, 19]. Methods to optimize selection on QTL to maximize a combination of short and longerterm responses have been derived $[3,5,16]$. Results showed that optimizing selection on QTL can result in greater response to selection within a pure line, although extra responses were limited, except for QTL with over-dominance. The objectives of this study were to extend these methods for simultaneous selection in two pure lines to maximize a combination of short and longer-term responses in crossbred performance, and to evaluate extra responses that can be achieved.

\section{METHODS}

\subsection{Population structure and genetic model}

A deterministic model was developed for a two-breed crossbreeding program consisting of purebred nucleus and multiplier populations for a male $(M)$ and a female $(F)$ line, along with a commercial crossbred population. Populations of infinite size with discrete generations were considered. All selection was within the purebred nucleus populations and based on data recorded in the nucleus only. Fractions of sires and dams selected each generation to produce nucleus replacements were $Q_{M s}$ and $Q_{M d}$ for the male line and $Q_{F s}$ and $Q_{F d}$ for the female line. Nucleus animals used as parents for the multiplier were a random sample of animals produced in the nucleus. All males from the male line multiplier and all females from the female line multiplier were used to produce commercial animals. Mating of sires and dams was at random at all levels. 
Table I. Summary of notation used for the model of selection on a QTL with two alleles ( $\mathrm{B}$ and $\mathrm{b}$ ) in generation $t$ in the male nucleus ${ }^{1}$.

\begin{tabular}{ccccc}
\hline $\begin{array}{c}\text { Geno- } \\
\text { type }\end{array}$ & Frequency $^{2}$ & Mean polygenic value $^{3}$ & $\begin{array}{c}\text { Mean genetic } \\
\text { value }\end{array}$ & $\begin{array}{c}\text { Mean breeding value, } \\
\text { deviated from } \\
\text { genotype } \mathrm{Bb}^{4}\end{array}$ \\
\hline $\mathrm{BB}$ & $p_{M, s, t} p_{M, d, t}$ & $\bar{u}_{M, B B, t}=A_{M, s, B, t}+A_{M, d, B, t}$ & $a+\bar{u}_{M, B B, t}$ & $\alpha_{t}+\bar{u}_{M, B B, t}-\bar{u}_{M, B b, t}$ \\
$\mathrm{Bb}$ & $p_{M, s, t}\left(1-p_{M, d, t}\right)$ & $\bar{u}_{M, B b, t}=A_{M, s, B, t}+A_{M, d, b, t}$ & $d+\bar{u}_{M, B b, t}$ & 0 \\
$\mathrm{bB}$ & $\left(1-p_{M, s, t}\right) p_{M, d, t}$ & $\bar{u}_{M, b B, t}=A_{M, s, b, t}+A_{M, d, B, t}$ & $d+\bar{u}_{M, b B, t}$ & $\bar{u}_{M, b B, t}-\bar{u}_{M, B b, t}$ \\
$\mathrm{bb}$ & $\left(1-p_{M, s, t}\right)\left(1-p_{M, d, t}\right)$ & $\bar{u}_{M, b b, t}=A_{M, s, b, t}+A_{M, d, b, t}$ & $-a+\bar{u}_{M, b b, t}$ & $-\alpha_{t}+\bar{u}_{M, b b, t}-\bar{u}_{M, B b, t}$ \\
\hline
\end{tabular}

${ }^{1}$ For parameters for the female nucleus, replace subscript $M$ by $F$.

${ }^{2} p_{M, s, t}$ and $p_{M, d, t}$ are frequencies of allele $\mathrm{B}$ among selected sires and dams that are used to produce generation $t$ in the male nucleus.

${ }^{3} \bar{u}_{M, m, t}$ is the mean polygenic breeding value of individuals of genotype $m$ in generation $t$ in the male nucleus, $A_{M, j, B, t}$ and $A_{M, j, b, t}$ are the mean polygenic values of gametes from sex $j$ that carry allele $\mathrm{B}$ or $\mathrm{b}$ and are used to produce generation $t$ in the male nucleus.

${ }^{4} \alpha_{t}$ is the QTL allele substitution effect in generation $t$, derived for the different selection strategies as described in the text.

Selection was for a trait controlled by a known QTL and additive infinitesimal polygenic effects [9]. The QTL had two alleles, B and b, with genotypic values equal to $a, d, d$, and $-a$ for genotypes $\mathrm{BB}, \mathrm{Bb}, \mathrm{bB}$, and bb (it was assumed that genotypes $\mathrm{Bb}$ and $\mathrm{bB}$, where the first letter refers to the paternal allele, could be distinguished). The variance of polygenic effects was assumed constant over generation, i.e. gametic phase disequilibrium among polygenes was ignored. All nucleus animals were genotyped for the QTL and phenotyped for the trait under selection. Effects at the QTL were assumed known without error.

Selection in each nucleus population was modeled as described in Dekkers and Chakraborty [6] for a single purebred population, by truncation selection across four distributions, one for each genotype (Fig. 1 of Dekkers and Chakraborty [6]). Further details and extension of this model to multiple alleles and multiple QTL are in Chakraborty et al. [3], but the notation of Dekkers and Chakraborty [6] for one QTL with two-alleles was used here for simplicity and presented in Table I. Given fractions selected from each distribution, equations (5), (6), and (7) of Dekkers and Chakraborty [6] were used to model changes in allele frequencies and polygenic means in each nucleus population from generation to generation. Polygenic variance was assumed to remain constant over generation, i.e. no Bulmer effect [2], but gametic phase 
disequilibrium between the QTL and polygenes was modeled, as described by Dekkers and Chakraborty [6].

\subsection{Selection objective and selection criteria}

Under random mating, and following the notation of Table I, the genetic level of crossbred progeny that originate from nucleus generation $t$ is:

$$
\begin{aligned}
G_{C t}= & 1 / 2\left[p_{M, s, t}+p_{M, d, t}+p_{F, s, t}+p_{F, d, t}-2\right] a \\
& \left.+1 / 2\left[p_{M, s, t}+p_{M, d, t}+p_{F, s, t}+p_{F, d, t}-\left(p_{M, s, t}+p_{M, d, t}\right)\left(p_{F, s, t}+p_{F, d, t}\right)\right] d\right\} \\
& +1 / 2\left[p_{M, s, t} A_{M, s, B, t}+\left(1-p_{M, s, t}\right) A_{M, s, B, t}+p_{M, d, t} A_{M, d, B, t}\right. \\
& \left.+\left(1-p_{M, d, t}\right) A_{M, d, B, t}\right] \\
& +1 / 2\left[p_{F, s, t} A_{F, s, B, t}+\left(1-p_{F, s, t}\right) A_{F, s, B, t}+p_{F, d, t} A_{F, d, B, t}\right. \\
& \left.+\left(1-p_{F, d, t}\right) A_{F, d, B, t}\right] .
\end{aligned}
$$

The general selection objective considered was to maximize cumulative discounted response (CDR) in crossbred performance over $T$ generations: $C D R_{T}=\sum_{t=1}^{T} w_{t} G_{t}$ with $w_{t}=1 /(1+r)^{t}$, where $r$ is the rate of interest per generation.

Five selection strategies were evaluated for their ability to increase $C D R_{T}$ over 5 or 10 generations based on purebred data in the male and female lines. Following Dekkers and Chakraborty [6], all strategies, including selection on phenotype, involved selection on a combination of the QTL and a polygenic estimated breeding value. Letting $\hat{\mathrm{u}}_{i, j, k, m, t}$ denote the polygenic breeding value estimate for individual $i$ of line $j(=M, F)$ of sex $k(=s, d)$ with genotype $m$ $(=\mathrm{BB}, \mathrm{Bb}, \mathrm{bB}$, or $\mathrm{bb})$ in generation $t$, the general selection criterion can be written as: $I_{i, j, k, m, t}=\theta_{j, k, m, t}+\left(\hat{\mathrm{u}}_{i, j, k, m, t}-\overline{\mathrm{u}}_{j, m, t}\right)$ where $\theta_{j, k, m, t}$ is a QTL value assigned to individuals of line $j$ of sex $k$ with genotype $m$ in generation $t$ and $\overline{\mathrm{u}}_{j, m, t}$ is the mean polygenic breeding value of individuals in line $j$ that have genotype $m$ in generation $t$. Based on this index, the following five selection criteria were defined for simultaneous selection within the male and female lines:

PHEN = selection on own phenotype. Implicit index values for the QTL, $\theta_{j, k, m, t}$, were derived as described in Dekkers and Chakraborty [6];

STD = standard QTL selection [6], with $\theta_{j, k, m, t}$ equal to the standard breeding values for the QTL for within-line selection (see below);

ALTSTD = alternate line standard QTL selection, with $\theta_{j, k, m, t}$ equal to standard breeding values for the QTL for crossbred selection; 
STEPOPT = stepwise optimal QTL selection, using QTL breeding values that maximize crossbred performance in the next generation, one generation at a time;

FULLOPT $=$ optimal QTL selection, maximizing $C D R_{T}$ over the planning horizon.

Breeding values for the QTL for strategies STD, ALTSTD, and STEPOPT were derived based on a QTL allele substitution effect $(\alpha)$ and polygenic means $(\overline{\mathrm{u}})$, using the following equation, after Dekkers and Chakraborty [6]: $\theta_{j, k, m, t}=n_{m} \alpha_{j, k, t}+\overline{\mathrm{u}}_{j, m, t}-\overline{\mathrm{u}}_{j, B b, t}$, where indicator variable $n_{m}$ is equal to +1 , 0,0 , and -1 for $m$ equal to $\mathrm{BB}, \mathrm{Bb}, \mathrm{bB}$, and $\mathrm{bb}$, respectively (see Tab. I) and where $\overline{\mathrm{u}}_{j, m, t}-\overline{\mathrm{u}}_{j, B b, t}$ quantifies gametic phase disequilibrium based on the mean polygenic difference of individuals of genotype $m\left(\overline{\mathrm{u}}_{j, m, t}\right)$ from the reference genotype $\mathrm{Bb}\left(\overline{\mathrm{u}}_{j, B b, t}\right)[6]$. For all three strategies, allele substitution effects were derived using the basic equation of Falconer and MacKay [9], i.e. $\alpha=a+(1-2 p) d$, where $p$ is the frequency of allele $\mathrm{B}$, but differed in the reference group for which the allele frequency was derived: the frequency among selection candidates from the same line was used for STD; the frequency among selection candidates of the opposite line for ALTSTD; and the frequency among selected parents in the opposite line for STEPOPT. The reference group for derivation of gametic phase disequilibrium was always based on selection candidates within the line for which the breeding values were derived. Thus, for selection in generation $t$ in the male line, noting that $p_{j, k, t}$ is defined as the frequency of B among individuals of sex $k$ in line $j$ that are used to produce generation $t$ for line $j$ (Tab. I) and, therefore, $\left(p_{j, s, t}+p_{j, d, t}\right) / 2$ is the frequency among selection candidates in line $j$, allele substitution effects were derived as follows:

$$
\begin{array}{ll}
\text { for STD: } & \alpha_{M, s, t}=\alpha_{M, d, t}=a+\left(1-p_{M, s, t}-p_{M, d, t}\right) d \\
\text { for ALTSTD: } & \alpha_{M, s, t}=\alpha_{M, d, t}=a+\left(1-p_{F, s, t}-p_{F, d, t}\right) d \\
\text { for STEPOPT: } & \alpha_{M, s, t}=\alpha_{M, d, t}=a+\left(1-p_{F, s, t+1}-p_{F, d, t+1}\right) d .
\end{array}
$$

Allele substitution effects for selection in the female line were similarly derived, by substituting $F$ for $M$ and $M$ for $F$.

The allele substitution effect for STEPOPT was based on an extension of Dekkers [4], who showed that QTL breeding values for selection of sires (dams) that maximize genetic progress from generation $t$ - 1 to $t$ within a purebred population can be derived from allele substitution effects that are based on allele frequencies among gametes produced by the selected group of dams (sires), rather than frequencies among selection candidates. In other words, 
the effect of a given QTL allele depends on the frequency of alleles that it will be combined with, rather than frequencies in the population from which it was selected. Extending this to maximizing performance of crossbred progeny, the frequency of $\mathrm{B}$ among alleles that combine with male nucleus alleles in the crossbred progeny that are derived from generation $t$ is equal to the frequency of $\mathrm{B}$ in the female nucleus in generation $t+1$, i.e. $1 / 2\left(p_{F, s, t+1}+p_{F, d, t+1}\right)$. Since QTL breeding values for the male line depend on allele frequencies among parents selected in the female line, which in turn, depend on allele frequencies among parents selected in the male line, solutions cannot be obtained analytically. An iterative procedure similar to that of Dekkers [4] was used, based on the appropriate sets of allele frequencies.

For full optimal QTL selection (FULLOPT), QTL breeding values $\theta_{j, k, m, t}$ were derived that maximized $C D R_{T}$ by extending the optimal control procedures of Dekkers and van Arendonk [5] and Chakraborty et al. [3] to simultaneous optimization of selection in male and female lines. Compared to the single-line problem of Chakraborty et al. [3], the crossbreeding problem required a separate set of variables for each line, including allele frequencies, mean polygenic breeding values, and Lagrange multipliers. This resulted in separate sets of equations of partial derivatives for each line (Eqs. (23) through (35) of Chakraborty et al. [3]). Partial derivatives were derived taking into account the altered objective function for $C D R_{T}$. This resulted in two sets of equations, similar to those represented in Figure 3 of Chakraborty et al. [3], which were solved simultaneously by a duplication of the iterative strategy of Chakraborty et al. [3]. Implementation of the extension was limited to the case of one QTL with two alleles.

Note that, in reference to equation (4) of Dekkers and Chakraborty [6], $\theta_{j, k, m, t}$ can also be written as the product of an index weight and the standard breeding value for the QTL, $\theta_{j, k, m, t}=b_{j, k, m, t} g_{k, m, t}$, where $g_{k, m, t}$ is the standard breeding value for the QTL, as is used for the STD selection strategy.

\subsection{Choice of parameters}

In the base situation, proportions selected in both nucleus populations were 0.1 for sires $\left(Q_{M s}=Q_{F s}=0.1\right)$ and 0.25 for dams $\left(Q_{M d}=Q_{F d}=0.25\right)$. Initial frequencies of allele B were 0.3 and 0.2 in the male and female line and starting populations were in gametic phase and Hardy Weinberg equilibrium. A range of additive and dominance effects at the QTL was evaluated for a trait with heritability 0.3: small $\left(a=0.5 \sigma_{p o l}\right)$, medium $\left(a=1 \sigma_{p o l}\right)$, or large $\left(a=2 \sigma_{p o l}\right)$ additive effects, where $\sigma_{p o l}$ is the polygenic standard deviation, and no $(d=0)$, 
partial $(d=1 / 2 a)$, complete $(d=a)$, or over-dominance $(d=1 / 1 / 2 a)$. Response in crossbred performance over ten generations with a discount rate of $10 \%$ per generation was evaluated.

Several parameters were changed from the base situation to further evaluate selection strategies. To evaluate the impact of equal starting frequencies in the two lines, starting frequencies were changed to 0.51 and 0.49 in the male and female lines (equal frequencies resulted in lack of convergence for FULLOPT). To evaluate the impact of differential selection intensities in the two lines, selected proportions were doubled for the male line to $Q_{M s}=0.2$ and $Q_{M d}=0.5$. And to evaluate the impact of length of the planning horizon, $C D R_{T}$ over 10 generations at a $0 \%$ discount rate and over 5 generations at a $30 \%$ discount rate were evaluated.

In all cases, responses were also compared to response from optimized selection in a single line (SLOPT), following Dekkers and Chakraborty [6]. To allow comparison to crossbreeding cases with different starting frequencies in the male and female lines, the base population was generated as a synthetic by crossing the two lines, which was followed by selection and random mating within the synthetic line.

\section{RESULTS}

\subsection{Base situation}

Table II shows $C D R_{T}$ for alternative QTL selection strategies relative to phenotypic selection for the base situation. For additive QTL and for QTL with partial dominance, all strategies, except FULLOPT, had lower $C D R_{T}$ than phenotypic selection, although differences were less than $3.6 \%$, and less than $2 \%$ for most cases. The advantage of FULLOPT over phenotypic selection was also small, less than $1 \%$ greater $C D R_{T}$, and implementing FULLOPT in the two-line program had no advantage over full optimal selection within a single line. Differences between programs were, however, greater for QTL with complete or over-dominance. With complete dominance, both STD and ALTSTD selection had lower response than phenotypic selection, up to $4 \%$ (Tab. II). For an over-dominant QTL, response was $0.8 \%$ lower for ALTSTD than for PHEN for a QTL of small effect, but $12.7 \%$ greater for a QTL with large effect. Response for STD was within 3\% of response to phenotypic selection for overdominant QTL. Full optimal selection had up to 3.3\% greater response than phenotypic selection for complete dominance and up to $21 \%$ greater response for overdominance. The difference in response between 
Table II. Extra (\%) cumulative discounted response (10 generations, 10\% interest) of QTL selection strategies over phenotypic selection for different degrees of dominance $(d)$ and QTL effects ( $a$ in polygenic s.d.) for the base situation.

\begin{tabular}{lcccc}
\hline & \multicolumn{5}{c}{ Degree of dominance } \\
\cline { 2 - 5 } Selection strategy & $d=0$ & $d=1 / 2 a$ & $d=a$ & $d=11 /{ }_{2} a$ \\
\hline Standard & -0.4 & -1.7 & -2.3 & -2.4 \\
Alternate standard & -0.4 & -1.6 & -2.0 & -0.8 \\
Stepwise optimal & -0.4 & -0.7 & -0.1 & 2.3 \\
Full optimal & 1.1 & 0.5 & 1.1 & 3.7 \\
Full optimal single line & 1.1 & 0.5 & 0.6 & 1.3 \\
\hline & & & $a=1$ & \\
Standard & -1.6 & -3.6 & -3.9 & -2.6 \\
Alternate standard & -1.6 & -3.5 & -3.6 & 1.4 \\
Stepwise optimal & -1.6 & -1.8 & 0.3 & 8.0 \\
Full optimal & 1.0 & 0.3 & 2.2 & 9.8 \\
Full optimal single line & 1.1 & 0.4 & 1.1 & 3.6 \\
\hline & & \multicolumn{4}{c}{$a=2$} \\
Standard & -1.3 & -3.2 & -3.1 & 1.5 \\
Alternate standard & -1.3 & -3.1 & -2.4 & 12.7 \\
Stepwise optimal & -1.3 & -2.3 & 1.1 & 20.9 \\
Full optimal & 0.8 & 0.1 & 3.3 & 21.0 \\
Full optimal single line & 0.9 & 0.2 & 1.8 & 9.7 \\
\hline
\end{tabular}

FULLOPT and STEPOPT was less than $2.2 \%$ for such QTL and both were substantially better than implementing optimal selection within a single line (up to $11.3 \%$ greater response for a large overdominant QTL). Results for complete and over-dominance are presented in further detail below.

\subsubsection{Complete dominance}

Trends in frequencies of the favorable QTL allele in the male and female lines for the six selection strategies are given in Figure 1 for QTL with complete dominance and a large additive effect of $2 \sigma_{\text {pol }}$. Trends were similar for 


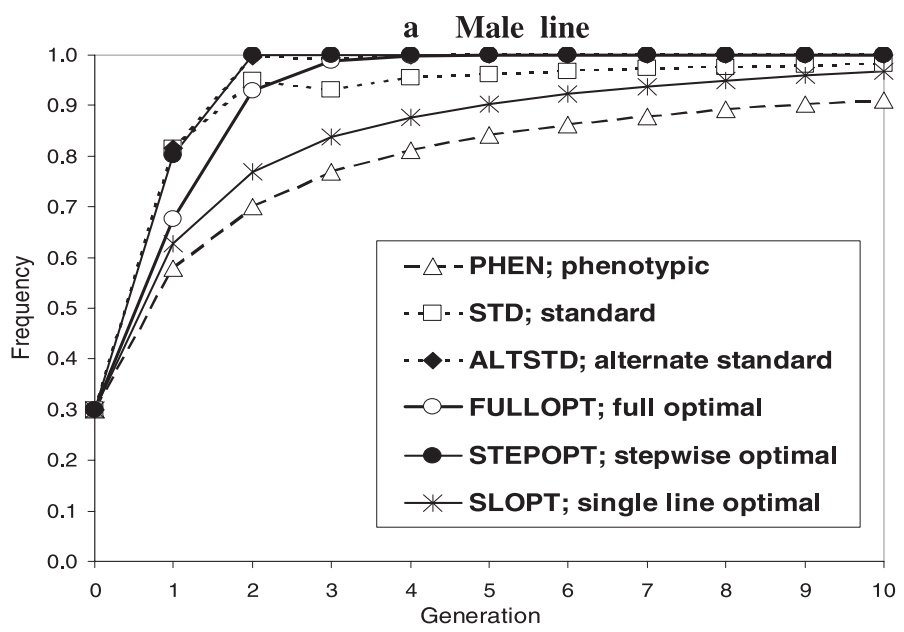

b Female line

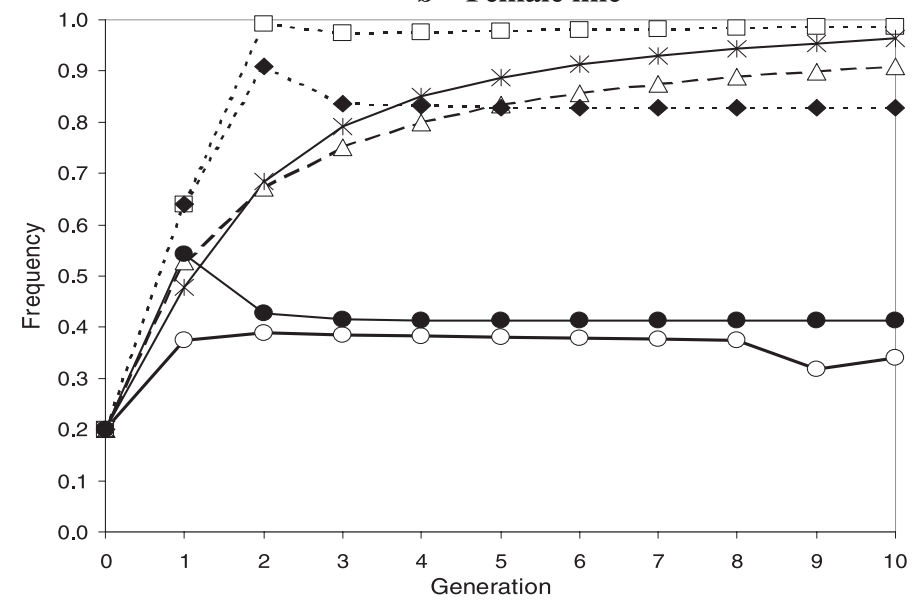

Figure 1. Frequency of the favorable allele in the (a) male line and (b) female line for different selection strategies for a QTL with complete dominance and a large additive effect (2 polygenic standard deviations), for the base situation parameters. Frequencies for single line optimal selection are among male (A) and female (B) gametes that contribute to a given generation.

the medium-sized QTL. For complete dominance, frequencies in the male line increased rapidly for all strategies, except for PHEN and SLOPT selection (Fig. 1a). Initial increases in frequency were slightly lower for FULLOPT than for STD, ALTSTD, and STEPOPT. Complete fixation was not reached for PHEN, STD, and SLOPT. Trends in female line frequencies (Fig. 1b) were similar to those for the male line for PHEN, STD, and SLOPT. For ALTSTD, 
frequency in the female line reached a constant value by generation 3. This was caused by fixation in the male line by generation 2 (Fig. 1a), which resulted in a zero allele substitution effect for the QTL in the female line $\left(\alpha_{F, s, t}=\alpha_{F, d, t}=a+\left(1-p_{M, s, t}-p_{M, d, t}\right) d=0\right.$ when $p_{M, s, t}=p_{M, d, t}=1$ and $d=a$ ). Note that female line frequencies declined in the first generation following fixation in the male line (from generation 2 to 3, Fig. 1b). This was caused by the negative gametic phase disequilibrium between the QTL and polygenes in the female line in generation 2 , which resulted in a negative emphasis on B alleles.

For the optimal selection strategies STEPOPT and FULLOPT, frequencies in the female line stabilized to 0.41 and 0.38 by generation 2 (Fig. 1b). By generation 2 the frequency of crossbred progeny with desirable QTL genotypes (BB, Bb, and $\mathrm{bB}$ ) was close to $100 \%$ for STEPOPT and FULLOPT (see Fig. 2a) and, as a result, there was no need to further select on the QTL in the female line, allowing all selection pressure to be applied to polygenes. Deviations from the stable frequency in the female line in generations 9 and 10 for FULLOPT (Fig. 1b) were caused by gametic phase disequilibrium. Similar trends were observed for a QTL of medium effect, except that female line frequencies stabilized at a lower level of 0.33 for both strategies. As demonstrated in Figure 2a, the frequency of crossbred progeny with desirable QTL genotypes increased to fixation for all strategies, but most rapidly for STD, ALTSTD, and STEPOPT.

Cumulative polygenic and total genetic gains in crossbreds for the large QTL are in Figure 3. Gains are expressed as a deviation from cumulative gains for phenotypic selection. Strategies that did not optimize selection over the entire planning horizon lost polygenic gain relative to phenotypic selection (Fig. 3a). Lost polygenic gains were greatest for STD and ALTSTD, and intermediated for STEPOPT. Polygenic gain was lost in initial generations because of the heavy emphasis on the QTL and could not be recovered in later generations, similar to what was observed by Dekkers and van Arendonk [5] for single line selection. Both strategies that optimized selection over the planning horizon (FULLOPT and SLOPT) achieved greater polygenic response than phenotypic selection. Extra polygenic response was greater for optimal selection in two lines than for optimal selection in a single line; FULLOPT was able to relax selection on the QTL in the female line after two generations (Fig. 1b), allowing it to maximize emphasis placed on polygenes. Similar trends were observed for the medium sized QTL, although differences between the six strategies were smaller. 

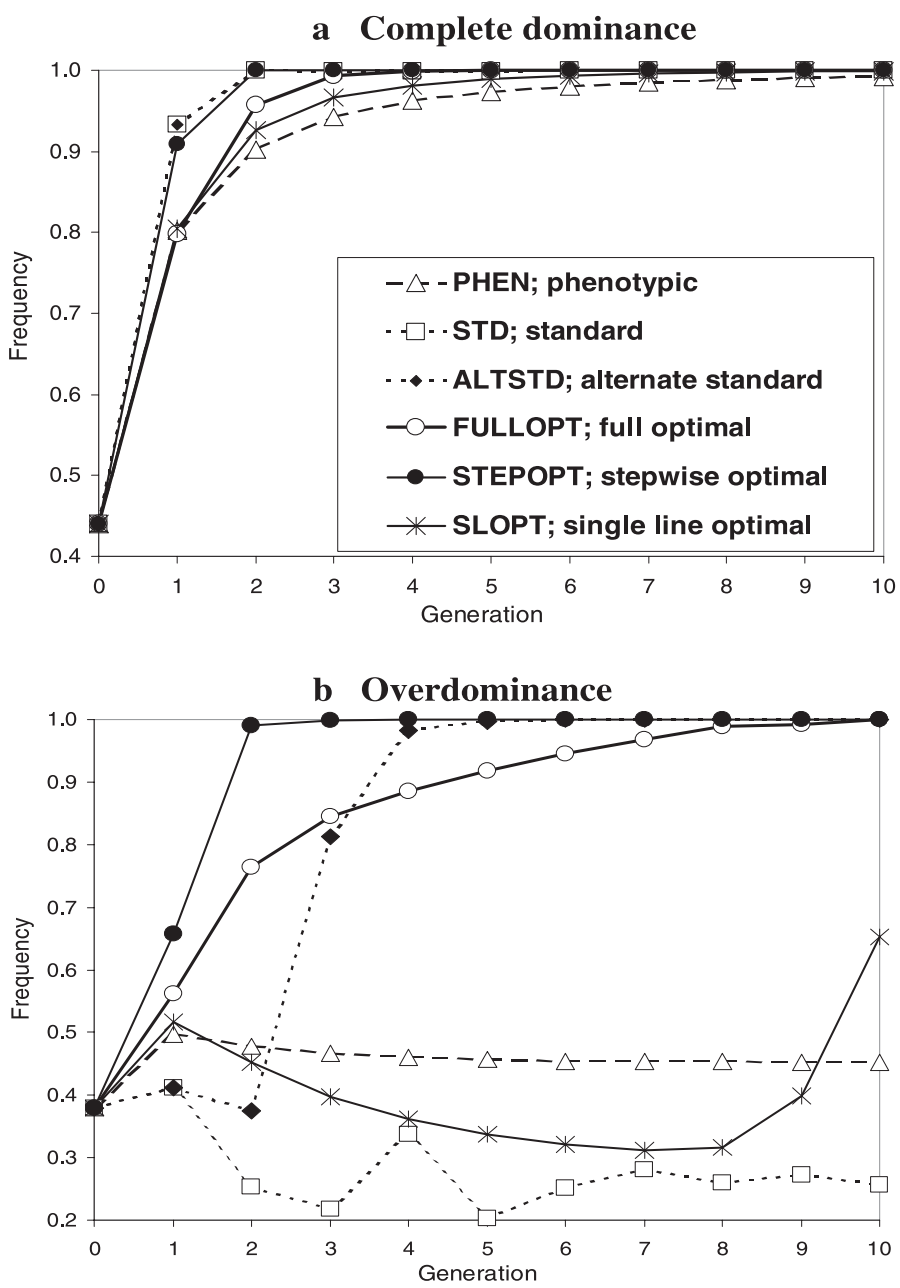

Figure 2. Frequency of desirable genotypes for different selection strategies for a QTL with (a) complete dominance (frequency of favorable homozygote plus heterozygotes) and (b) overdominance (frequency of heterozygotes) and a large additive effect ( 2 polygenic standard deviations), for the base situation parameters.

Lost polygenic responses for STD, ALTSTD, and STEPOPT were offset by greater gains in the QTL during the initial generations, resulting in greater total genetic gain after one generation (Fig. 3b). Strategy STEPOPT achieved the greatest total genetic response in generation 1, as expected, but was closely followed by STD and ALTSTD. Cumulative total response for STD and ATLSTD, however, dropped below response to phenotypic selection after a few generations, as the frequency of desirable genotypes for phenotypic 
a Polygenic response

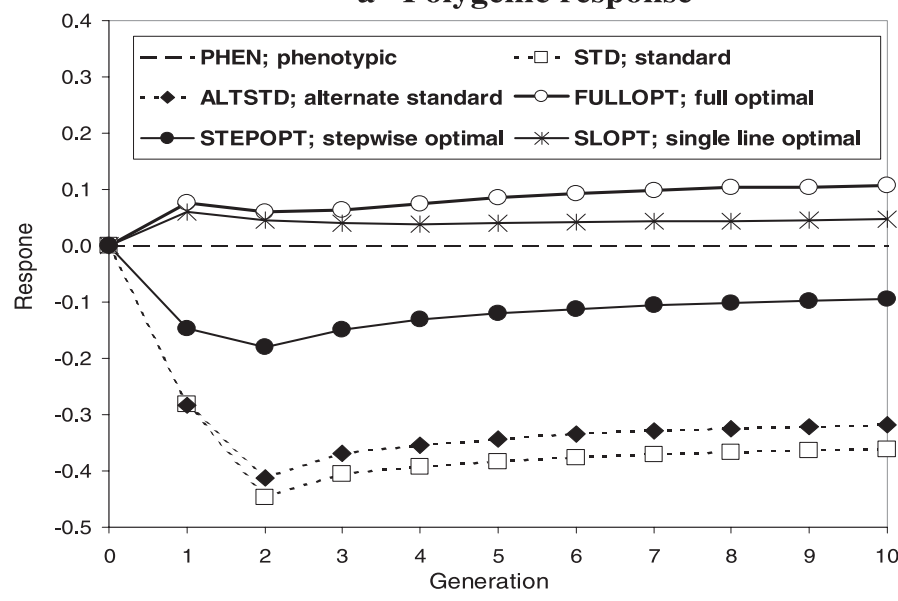

b Total genetic response

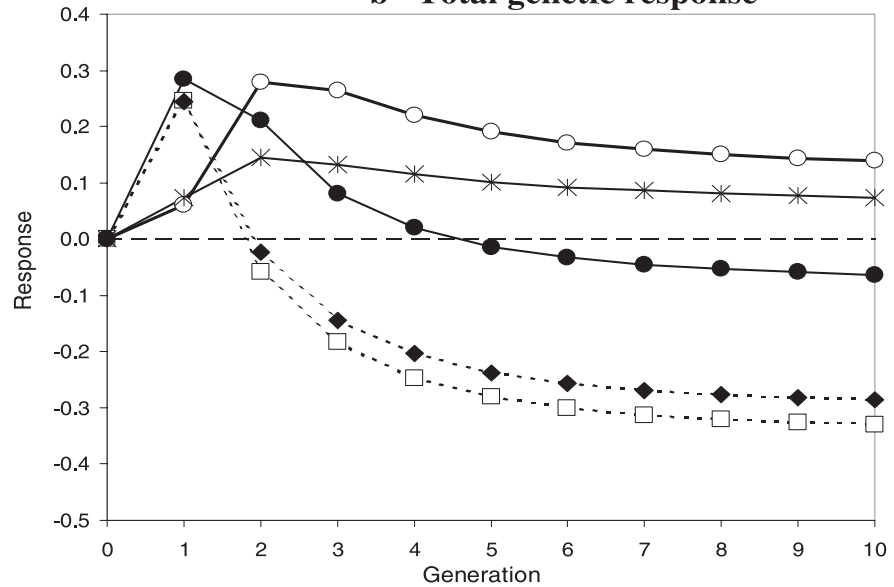

Figure 3. Cumulative polygenic (a) and total genetic (b) response to different selection strategies for a QTL with complete dominance and a large additive effect (2 polygenic standard deviations), as a deviation from phenotypic selection, for the base situation parameters.

selection moved toward fixation (Fig. 2a). Cumulative response from STEPOPT dropped below response from phenotypic selection by generation five. Strategy FULLOPT had the greatest cumulative response by generation 2 and thereafter (Fig. 3b), demonstrating its ability to balance response in the QTL and polygenes. Optimal selection in two lines resulted in greater cumulative responses than optimal selection in a single line in generations 2 and later. 

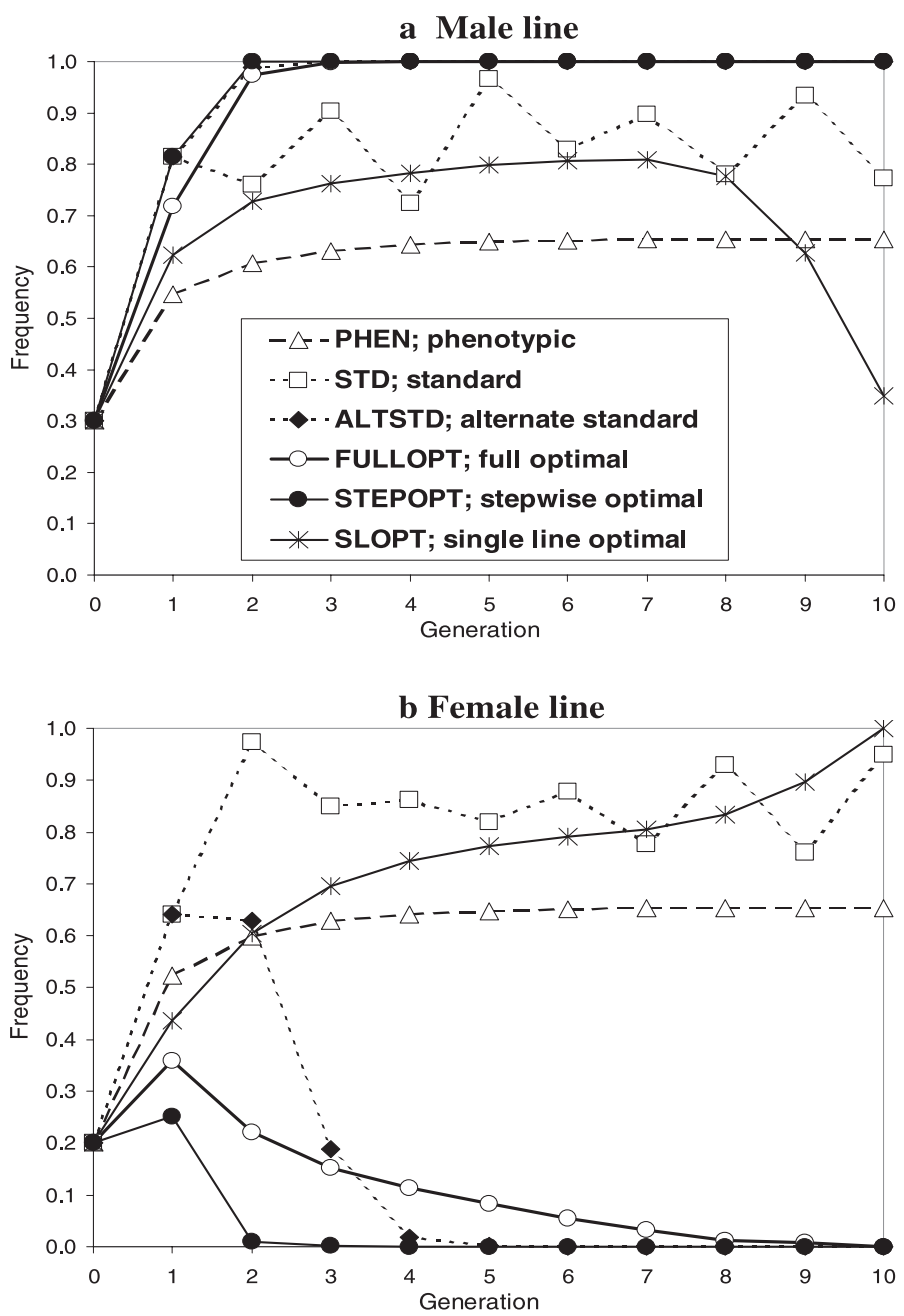

Figure 4. Frequency of the favorable allele in the (a) male line and (b) female line for different selection strategies for a QTL with overdominance and a large additive effect ( 2 polygenic standard deviations), for the base situation parameters. Frequencies for single line optimal selection are among male (A) and female (B) gametes that contribute to a given generation.

\subsubsection{Overdominance}

Trends in allele frequencies in the male and female lines for a large over-dominant QTL are in Figure 4. The optimal strategies STEPOPT and FULLOPT resulted in fixation of alternate QTL alleles in the male and the female line, which led to a rapid increase in the frequency of the most desirable 
genotype, heterozygotes (Fig. 2b). Fixation of alleles was more rapid for STEPOPT than for FULLOPT. Strategy ALTOPT also resulted in ultimate fixation of alternate alleles but the frequency of allele B initially increased in both lines (Fig. 4). Allele substitution effects with ALTSTD are determined by frequencies in the opposite line and change from positive to negative at a frequency of 0.83 , which is were the substitution effect is zero for an overdominant QTL with $d=1 \frac{1}{2} 2 a$. Thus, once the frequency in the male line rose to above 0.83 in generation 1, the QTL allele substitution effect became negative for the female line and the frequency in the female line decreased.

For STD, allele frequencies in the two lines oscillated around the equilibrium frequency of 0.83 (Fig. 4). Frequencies among male and female gametes also approached the equilibrium frequency under optimal selection in a single line but diverged in the final two generations to increase the frequency of heterozygotes (Fig. 2b). Frequencies for PHEN asymptoted to a frequency of 0.65 .

Frequencies of the desired QTL genotype, heterozygotes, rapidly increased to $100 \%$ for STEPOPT (Fig. 2b) and, with a 2-generation delay, also for ALTSTD. The increase in frequency of heterozygotes was more gradual for FULLOPT. Frequency of heterozygotes was less than $50 \%$ for phenotypic, STD, and SLOPT, but increased for the latter in the final generations.

Cumulative polygenic responses for the overdominant QTL are in Figure 5. In contrast to the QTL with complete dominance, the advantage of most strategies over phenotypic selection increased over generations. This was caused by the continuous implicit selection on heterozygotes with phenotypic selection because of their greater phenotypic value, but with no effect on QTL frequencies (Figs. 2b and 4). In contrast, strategies that used QTL information seized to select on the QTL once it was fixed or when the equilibrium frequency was reached. An exception was STD, which oscillated around the equilibrium frequency (Fig. 4) and, therefore, also continued to put emphasis on the QTL.

Among the optimal strategies, SLOPT obtained the greatest cumulative polygenic gains (Fig. 5a), followed by FULLOPT. The ability for FULLOPT to increase the number of heterozygotes by divergent selection on the QTL, however, resulted in a substantially greater cumulative total response than SLOPT (Fig. 5b). The difference in total response between FULLOPT and STEPOPT was small (Fig. 5b), despite a substantially greater polygenic response for FULLOPT (Fig. 5a). This was, however, partially offset by a slightly greater frequency of heterozygotes for STEPOPT (Fig. 2b). 


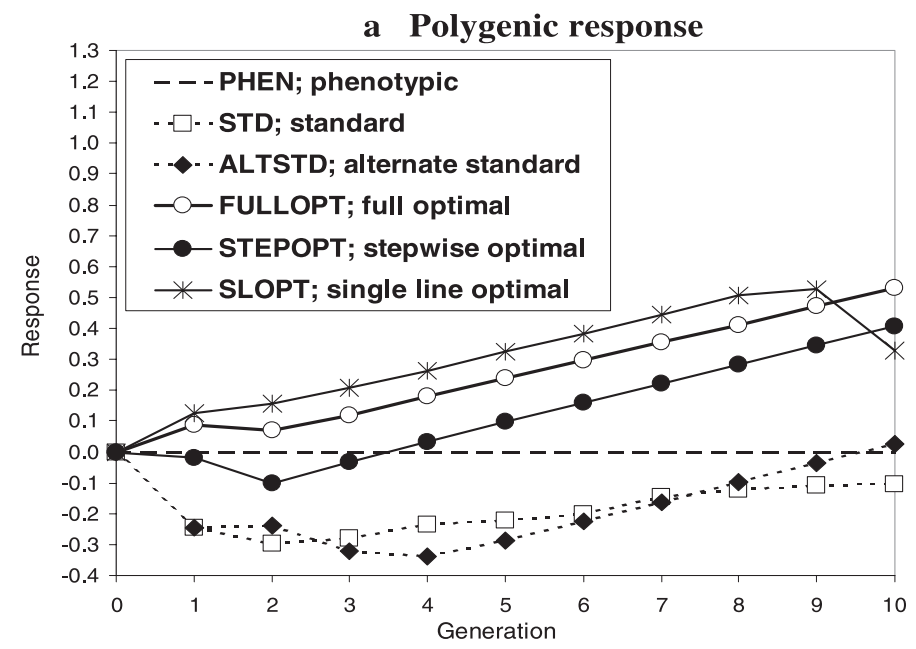

b Total genetic response

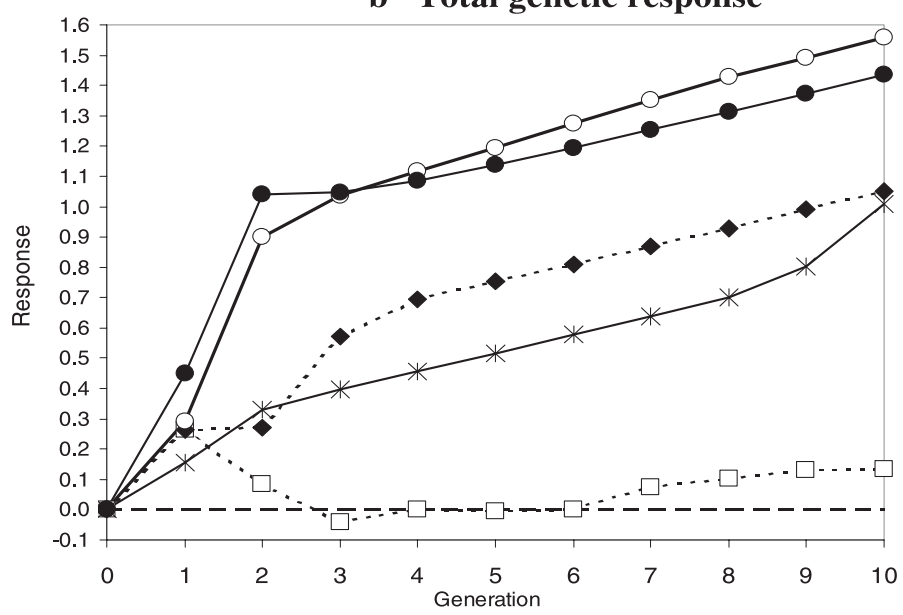

Figure 5. Cumulative polygenic (a) and total genetic (b) response to different selection strategies for a QTL with overdominance and a large additive effect (2 polygenic standard deviations), as a deviation from phenotypic selection, for the base situation parameters.

\subsection{Alternative parameters}

\subsubsection{Initial frequencies}

Intermediate starting frequencies for the favorable QTL allele $(0.51$ and 0.49 in the male and female line) had limited impact on differences in $C D R_{T}$ between selection strategies (Tab. III). The main difference was that relative 
Table III. Extra (\%) cumulative discounted response (10 generations, 10\% interest) of QTL selection strategies over phenotypic selection for different degrees of dominance $(d)$ and QTL effects ( $a$ in polygenic s.d.) for alternative sets of parameters.

\begin{tabular}{|c|c|c|c|c|c|c|c|c|}
\hline \multirow[b]{2}{*}{ Selection strategy } & \multicolumn{2}{|c|}{$\begin{array}{l}\text { Starting frequencies } \\
0.51 \text { and } 0.49 \text { in } \\
\text { male and female } \\
\text { line }\end{array}$} & \multicolumn{2}{|c|}{$\begin{array}{l}\text { Male line selected } \\
\text { proportions } \\
\text { doubled to } 0.2 \\
\text { and } 0.5\end{array}$} & \multicolumn{2}{|c|}{$\begin{array}{l}\text { Long planning } \\
\text { horizon } \\
\text { (10 generations } \\
0 \% \text { interest) }\end{array}$} & \multicolumn{2}{|c|}{$\begin{array}{l}\text { Short planning } \\
\text { horizon } \\
\text { ( } 5 \text { generations } \\
30 \% \text { interest) }\end{array}$} \\
\hline & $d=a$ & $d=1 \frac{1}{2} a$ & $d=a$ & $d=1 \frac{1}{2} a$ & $d=a$ & $d=1 / 12 a$ & $d=a$ & $d=1 \frac{1}{2} a$ \\
\hline & \multicolumn{8}{|c|}{$a=0.5$} \\
\hline Standard & -0.9 & -0.4 & -2.3 & -2.3 & -2.4 & -2.2 & -1.9 & -3.1 \\
\hline Alternate standard & -1.0 & -0.2 & -2.2 & -2.0 & -2.0 & -0.6 & -1.4 & -1.4 \\
\hline Stepwise optimal & 0.1 & 1.9 & -0.5 & 1.3 & -0.4 & 1.9 & 1.6 & 4.1 \\
\hline Full optimal & 0.5 & 2.6 & 0.8 & 3.2 & 1.0 & 3.6 & 2.5 & 5.4 \\
\hline \multirow[t]{2}{*}{ Full optimal single line } & 0.5 & 1.3 & & & 0.5 & 1.3 & 1.6 & 2.0 \\
\hline & \multicolumn{8}{|c|}{$a=1$} \\
\hline Standard & -2.8 & -2.2 & -3.9 & -2.5 & -3.9 & -2.4 & -3.6 & -3.6 \\
\hline Alternate standard & -2.8 & -0.5 & -3.9 & -1.9 & -3.6 & 1.6 & -3.2 & 0.2 \\
\hline Stepwise optimal & 0.4 & 6.6 & -0.7 & 4.5 & -0.1 & 7.4 & 2.8 & 11.9 \\
\hline Full optimal & 1.2 & 8.7 & 1.6 & 8.4 & 2.0 & 9.4 & 4.4 & 13.2 \\
\hline \multirow[t]{2}{*}{ Full optimal single line } & 1.1 & 3.9 & & & 1.0 & 3.6 & 2.5 & 4.6 \\
\hline & \multicolumn{8}{|c|}{$a=2$} \\
\hline Standard & -4.2 & -2.2 & -3.0 & 0.5 & -3.4 & 1.3 & -1.1 & 2.9 \\
\hline Alternate standard & -4.2 & 6.3 & -3.0 & 3.9 & -2.8 & 12.6 & -0.3 & 13.1 \\
\hline Stepwise optimal & -1.0 & 24.0 & 0.2 & 16.1 & 0.5 & 19.9 & 4.4 & 26.1 \\
\hline Full optimal & 2.3 & 24.0 & 2.8 & 17.5 & 2.9 & 20.9 & 6.1 & 26.4 \\
\hline Full optimal single line & 1.9 & 12.5 & & & 1.6 & 9.8 & 3.9 & 12.2 \\
\hline
\end{tabular}

responses to ALTSTD tended to be reduced relative to the base situation, in particular for the large QTL with over-dominance. Although alternate QTL alleles were ultimately fixed in both lines under this strategy, frequencies of the favorable allele increased to 0.99 and 0.98 in the male and female line in generation 1. This required much additional selection pressure on the QTL over generations, which resulted in lower polygenic response. Frequencies for STD again oscillated around the equilibrium frequency of 0.83 (results not shown). 


\subsubsection{Differential selection intensities}

Doubling selected proportions in the male line had limited impact on differences in $C D R_{T}$ between selection strategies for a QTL with complete dominance (Tab. III) but reduced the benefit of stepwise and full optimal selection strategies over phenotypic selection for the over-dominant QTL, compared to the base situation (Tab. II). Strategy ALTSTD also had lower $C D R_{T}$ relative to phenotypic selection when selection intensities in the male line were reduced.

For these parameters, the favorable allele tended to be fixed in the female rather than in the male line for the optimal selection strategies and for ALTSTD for both complete and over-dominance (Tab. IV). For FULLOPT, the reason for this is that the greater selection intensities in the female line allowed for more opportunities for large changes in allele frequencies than were present in the male line. For complete dominance and overdominant QTL of small effect, however, the favorable allele tended to be fixed in the male line (Tab. IV). The same occurred for STEPOPT for the medium-sized QTL. For ALTSTD, selection on a small overdominant QTL resulted in ultimate divergence of the QTL but at a very slow rate (Tab. IV).

\subsubsection{Planning horizon}

Increasing the length of the planning horizon by reducing the discount rate to zero had limited impact on responses between strategies (Tabs. II and III). For a short planning horizon (five generations and a 30\% discount rate), however, the benefit of the optimal selection strategies was greater than for the long time horizon (Tab. III). Extra $C D R_{T}$ over phenotypic selection was up to $6 \%$ for complete dominance and up to $27 \%$ for over-dominance for FULLOPT. Extra responses for STEPOPT were within 2\% of those for FULLOPT. Strategy FULLOPT resulted in a rapid increase in the frequency of desirable genotypes without sacrificing much polygenic response relative to phenotypic selection (Tab. V). For complete dominance, no selection pressure was placed on the QTL in the female line, except for the first generation, which allowed increased polygenic gain in that line.

\section{DISCUSSION AND CONCLUSIONS}

\subsection{Selection on QTL for crossbred performance}

This study demonstrates that strategic use of non-additive QTL enables selection for crossbred performance based on purebred data. Compared to 
Table IV. Frequencies of the favorable QTL allele in the male and female line (male/female) for alternate standard (ALTSTD), stepwise optimal (STEPOPT), and full optimal (FULLOPT) selection for cumulative discounted response (10 generations, $10 \%$ interest) on a QTL with complete or over-dominance and different additive effects, with doubled selected proportions of sires and dams of 0.2 and 0.5 in the male line.

\begin{tabular}{lcccccc}
\hline \multirow{2}{*}{$\begin{array}{l}\text { Selection } \\
\text { strategy }\end{array}$} & \multicolumn{3}{c}{ Complete dominance } \\
\cline { 2 - 7 } & 2 & 5 & 10 & 2 & 5 & 10 \\
\hline & & \multicolumn{5}{c}{$a=0.5$} \\
ALTSTD & $0.71 / 0.68$ & $0.84 / 0.83$ & $0.91 / 0.92$ & $0.72 / 0.66$ & $0.83 / 0.70$ & $0.98 / 0.46$ \\
STEPOPT & $0.65 / 0.56$ & $0.84 / 0.73$ & $0.95 / 0.82$ & $0.73 / 0.42$ & $1.00 / 0.15$ & $1.00 / 0.02$ \\
FULLOPT & $0.60 / 0.35$ & $0.86 / 0.42$ & $0.99 / 0.44$ & $0.68 / 0.22$ & $0.96 / 0.12$ & $1.00 / 0.02$ \\
\hline & & \multicolumn{7}{c}{$a=1$} \\
ALTSTD & $0.85 / 0.89$ & $0.88 / 0.95$ & $0.90 / 0.99$ & $0.83 / 0.85$ & $0.77 / 0.84$ & $0.31 / 1.00$ \\
STEPOPT & $0.71 / 0.79$ & $0.70 / 0.99$ & $0.70 / 1.00$ & $0.91 / 0.39$ & $1.00 / 0.03$ & $1.00 / 0.00$ \\
FULLOPT & $0.45 / 0.71$ & $0.45 / 0.98$ & $0.45 / 1.00$ & $0.27 / 0.80$ & $0.09 / 1.00$ & $0.01 / 1.00$ \\
\hline & & \multicolumn{7}{c}{$a=2$} & & \\
ALTSTD & $0.95 / 0.99$ & $0.92 / 0.99$ & $0.93 / 0.99$ & $0.93 / 0.96$ & $0.69 / 0.90$ & $0.00 / 1.00$ \\
STEPOPT & $0.58 / 1.00$ & $0.56 / 1.00$ & $0.56 / 1.00$ & $0.31 / 1.00$ & $0.00 / 1.00$ & $0.00 / 1.00$ \\
FULLOPT & $0.44 / 0.89$ & $0.42 / 1.00$ & $0.42 / 1.00$ & $0.50 / 0.80$ & $0.19 / 1.00$ & $0.00 / 1.00$ \\
\hline
\end{tabular}

phenotypic selection, limited benefits were obtained for QTL with partial dominance. Extra responses, evaluated as cumulative discounted response, combining short- and long-term responses, were slightly greater for complete dominance but still less than 3.5\% (Tabs. II and III). For overdominant QTL $\left(d=1 \frac{1}{2} a\right)$, extra responses over phenotypic selection that were obtained with optimal QTL selection increased nearly linear with the magnitude of the additive effect of the QTL and were up to 6, 13, and 26\% greater for QTL with additive effects of $1 / 2,1$, and $2 \sigma_{\mathrm{pol}}$. At a frequency of 0.25 and polygenic EBV based on phenotype for a trait with heritability 0.3 , this represents QTL that explain 27,59, and $85 \%$ of the purebred genetic variance. Although these QTL effects may be unrealistic, similar results may be obtained from selection on multiple QTL that jointly explain such a proportion of genetic variance.

While differences in response of QTL selection strategies with phenotypic selection depend on heritability, relative differences among QTL selection strategies depend only on the magnitude of the QTL relative to the standard deviation of polygenic $\operatorname{EBV}\left(\sigma_{E B V}=\right.$ accuracy $\left.* \sigma_{p o l}\right)$, as demonstrated 
Table V. QTL allele and desirable genotype frequencies, and polygenic and genetic means for crossbred progeny for phenotypic selection and for full optimal selection for cumulative discounted response ( 5 generations, $30 \%$ interest) on a large effect QTL ( $a=2$ polygenic standard deviations) with complete or over-dominance.

\begin{tabular}{|c|c|c|c|c|c|c|c|c|}
\hline \multirow[b]{2}{*}{$\begin{array}{l}\text { Gene- } \\
\text { ration }\end{array}$} & \multicolumn{4}{|c|}{ Complete dominance } & \multicolumn{4}{|c|}{ Over-dominance } \\
\hline & $\begin{array}{l}\text { Male/female } \\
\text { frequency }^{1}\end{array}$ & $\begin{array}{l}\mathrm{BB}+\mathrm{Bb} \\
\text { frequency }\end{array}$ & $\begin{array}{c}\text { Polygenic } \\
\text { mean }\end{array}$ & $\begin{array}{l}\text { Genetic } \\
\text { mean }\end{array}$ & $\begin{array}{l}\text { Male/female } \\
\text { frequency }\end{array}$ & $\begin{array}{c}\mathrm{Bb} \\
\text { frequency }\end{array}$ & $\begin{array}{c}\text { Polygen } \\
\text { mean }\end{array}$ & $\begin{array}{l}\text { Genetic } \\
\text { mean }\end{array}$ \\
\hline & \multicolumn{8}{|c|}{ Phenotypic selection } \\
\hline 0 & $0.30 / 0.20$ & 0.44 & 0 & 0 & $0.30 / 0.20$ & 0.38 & 0 & 0 \\
\hline 1 & $0.58 / 0.53$ & 0.80 & 0.59 & 2.03 & $0.55 / 0.52$ & 0.50 & 0.55 & 2.04 \\
\hline 2 & $0.70 / 0.67$ & 0.90 & 1.34 & 3.19 & $0.61 / 0.60$ & 0.48 & 1.28 & 2.99 \\
\hline 3 & $0.77 / 0.75$ & 0.94 & 2.14 & 4.15 & $0.63 / 0.63$ & 0.47 & 2.04 & 3.82 \\
\hline 4 & $0.81 / 0.80$ & 0.96 & 2.95 & 5.04 & $0.64 / 0.64$ & 0.46 & 2.80 & 4.61 \\
\hline \multirow[t]{2}{*}{5} & $0.84 / 0.83$ & 0.97 & 3.77 & 5.90 & $0.65 / 0.65$ & 0.46 & 3.57 & 5.39 \\
\hline & \multicolumn{8}{|c|}{ Full optimal selection } \\
\hline 0 & $0.30 / 0.20$ & 0.44 & 0 & 0 & $0.30 / 0.20$ & 0.38 & 0 & 0 \\
\hline 1 & $0.75 / 0.41$ & 0.85 & 0.59 & 2.23 & $0.79 / 0.25$ & 0.65 & 0.58 & 2.44 \\
\hline 2 & $1.00 / 0.32$ & 1.00 & 1.29 & 3.53 & $1.00 / 0.05$ & 0.95 & 1.25 & 4.06 \\
\hline 3 & $1.00 / 0.31$ & 1.00 & 2.12 & 4.36 & $1.00 / 0.01$ & 0.99 & 2.06 & 4.91 \\
\hline 4 & $1.00 / 0.31$ & 1.00 & 2.95 & 5.19 & $1.00 / 0.01$ & 0.99 & 2.89 & 5.74 \\
\hline 5 & $1.00 / 0.31$ & 1.00 & 3.78 & 6.02 & $1.00 / 0.00$ & 1.00 & 3.72 & 6.57 \\
\hline
\end{tabular}

${ }^{1}$ Frequency of the favorable QTL allele among male line and female line individuals that contribute to generation $t$ of crossbreds.

by Dekkers and van Arendonk [5]. Thus, differences between QTL selection strategies for the small, medium, and large QTL with $a=1 / 2,1$, and $2 \sigma_{\text {pol }}$ for EBV based on own phenotype for a trait with heritability 0.3 (thus accuracy $=\sqrt{0.3}$ ) apply to QTL with additive effects on the EBV scale of $a=0.91,1.83$, and $3.65 \sigma_{E B V}$.

Results presented herein apply to an identified, rather than a marked, QTL with known effects. Similar results will, however, hold for selection on markers that are in strong and persistent population-wide gametic phase disequilibrium with the QTL.

\subsubsection{Nature of extra responses from optimal selection}

The majority of the extra crossbred response obtained by optimizing selection across the planning horizon (FULLOPT) were also obtained by optimizing 
one generation at a time (STEPOPT); extra responses from STEPOPT were within $2 \%$ of those from FULLOPT for nearly all scenarios evaluated (Tabs. II and III). Strategy STEPOPT uses QTL allele substitution effects that are based on allele frequencies among selected individuals in the opposite line, which are the expected frequencies among potential mates in the crossbreeding phase. In concept, this is equivalent to the allele substitution effects that are used to derive single generation optimal breeding values derived by Dekkers [4] for genetic improvement within a population. For maximization of crossbred performance, however, selected mates originate from the opposite line, rather than from the same population. Because selection in one line depends on selection in the other line, allele substitution effects under STEPOPT must be derived numerically, similar to Dekkers [4].

Greater crossbred performance from FULLOPT and STEPOPT resulted not only from greater QTL response, but also from greater polygenic responses (Figs. 3, 5 and Tab. IV). This is most clearly demonstrated for QTL with complete dominance, where emphasis on the QTL was relaxed in one of the lines, as the favorable allele was moved to fixation in the other line (Fig. 1b). This allowed rapid increases in the frequency of desirable genotypes while maximizing polygenic response. Which line was chosen for fixation of the favorable allele depended on starting frequencies and selection intensities in the two lines, and on the effect of the QTL. For example, with equal selection intensities in both lines, the QTL was fixed in the line with the highest starting frequency (Figs. 1 and 4). However, when selection intensities were reduced in the line with the higher starting frequency, fixation in the other line was more beneficial, except when the QTL effect was small (Tab. IV). The reason for the latter is that with small QTL, rapid changes in QTL frequencies are less important than maximizing polygenic response. The same three factors determined in which line the favorable allele was fixed for a QTL with overdominance (Fig. 4 and Tab. IV). Choice of the correct line to fix the favorable allele in may not have a large impact on results, however, except when starting frequencies differ widely. In practice, other factors, including marketing, costs of genotyping, and direct and pleiotropic QTL effects, which may make a certain genotype more or less suitable for a male than a female line, will play a role in determining which line to fix the favorable allele in.

Full optimal selection generally minimized the extra effort required to move QTL to their optimal frequencies by gradual and unidirectional increases or decreases in frequencies in each of the two lines, such that polygenic response was maximized. There were, however, some exceptions. For example, with a large overdominant QTL, the frequency in the female line initially increased 
from 0.2 to 0.35 in generation 1 , followed by a gradual decrease (Fig. 2b). Notice that STEPOPT also had a slight increase in frequency in generation 1 in the female line, but only to 0.25 . Although these increases resulted in some loss in polygenic response, both in generation 1 and in later generations because the increase in frequency had to be reversed, these losses were more than offset by greater responses obtained from the QTL. To illustrate the latter, with the allele frequency in generation 1 for FULLOPT being 0.7 , an increase in the frequency in the female line to 0.35 resulted in a $0.12 \sigma_{\text {pol }}$ greater mean QTL genotype value than maintaining the frequency at 0.2 . Although this resulted in some lost polygenic response, these losses were only $0.03 \sigma_{\text {pol }}$ in generation 1 , with no additional loss in generation 2 because the negative disequilibrium that was established between the QTL and polygenes in generation 1 led to an automatic decline in frequency in generation 2. Even accumulated over generations, the short-term gain in QTL response more than offset the permanent loss of polygenic response.

\subsubsection{Standard selection strategies on $Q T L$}

Both standard strategies of selection on the QTL, STD and ALTSTD, resulted in substantially lower responses than the optimal strategies (Tabs. II and III) and often had lower responses than phenotypic selection. Both STD and ALTSTD use QTL allele substitution effects derived from QTL frequencies among selection candidates, rather than frequencies among selected individuals, which are used for STEPOPT. For STD selection, which uses frequencies from the individual's own line, this results in selection toward the same frequency in both lines; fixation of the favorable allele for QTL with partial or complete dominance and toward the equilibrium frequency for overdominant QTL. For overdominant QTL, the path to fixation tended to be erratic for STD, with frequencies oscillating around the equilibrium because of the change in sign of the allele substitution effect across the point of equilibrium (Fig. 4). Strategy ALTSTD uses frequencies in the opposite line to compute allele substitution effects. This is similar to STEPOPT, which also uses frequencies from the opposite line, except frequencies used for STEPOPT are among selected individuals, rather than based on all selection candidates. Use of frequencies from the opposite line, as in ALTSTD, instead of frequencies from the same line, as in STD, has limited impact for QTL with partial or complete dominance; the QTL is moved toward fixation in both lines for both STD and ALTSTD, although the rate of fixation may differ between the two strategies. For overdominant QTL, however, ALTSTD resulted in divergence 
of allele frequencies in the two lines, as illustrated in Figure 4 and Table IV. When the two lines diverge for this strategy, however, depends on simultaneous changes in frequencies for each line relative to the equilibrium frequency point and is not driven by maximizing response to selection. For example, for the small and medium-sized QTL in Table IV, frequencies in both lines ultimately diverged but at a rate determined by the combination of frequencies in the two lines relative to the equilibrium frequency of 0.83 .

The lower responses for STD and ALTSTD compared to FULLOPT are the result of a combination of factors, depending on the level of dominance at the QTL. In all cases, STD and ALTSTD resulted in lower polygenic response in the initial generations because of greater emphasis on the QTL. Similar to what has been observed for single line selection [5], polygenic response lost in early generations was not completely recovered in later generations because of the non-linear relationship between selection emphasis and response to selection. This explains the main portion of the lower responses for STD and ALTSTD compared to phenotypic selection. For QTL with complete dominance and overdominance, two additional factors led to lower responses for STD and ALTSTD relative to optimal selection strategies, in particular for over-dominant QTL: (1) lower frequencies of the desirable genotype because of lack of (for STD) or delayed (for ALTSTD) divergence of the two lines, and (2) changes in the direction of selection on the QTL, which wasted selection effort that could have been placed on the polygenes, i.e. initial increases in frequency of the QTL followed by a decline for ALTSTD (Fig. 4b), and the oscillating behavior of frequencies for STD (Fig. 4).

\subsection{Degree of dominance at QTL}

Results showed limited benefits from optimizing selection for QTL with partial or even complete dominance (Tabs. II and III). With partial dominance the optimal strategy is to maximize the favorable QTL allele in both the male and the female line, although the rate at which fixation occurs may still benefit from optimization, similar to single line selection [6]. This may be of particular importance with simultaneous selection on multiple QTL. Thus, opportunities for optimal selection to capitalize on selection for increased heterosis will be limited unless QTL with overdominance exist.

Several theories exist to explain the nature of heterotic effects that are observed for quantitative traits, including the dominance and over-dominance models of heterosis [14]. Whereas heterosis results from heterozygote superiority under the overdominance model, under the dominance model, heterotic 
effects are due to multiple genes with partial or complete dominance for which favorable alleles are in higher frequency in one line for some genes and for other genes in the other line. The ability to distinguish between these two modes of action is difficult by conventional quantitative genetic means and requires adequate population designs, even for the study of dominance at the individual gene level based on molecular information. For example, many recent QTL studies, in particular in breed crosses, have found overdominant QTL $[8,15]$. Most of these may, however, represent pseudo overdominance effects that are caused by linked QTL in repulsion phase rather real overdominance at a single QTL [14]. If their linkage and linkage disequilibrium is tight, however, pseudo overdominance effects are expected to persist for some time in the population and there would be benefit to divergent selection to capitalize on short-term benefits. Sufficient emphasis would need to be put on identification of and subsequent selection on recombinants that break the repulsion phase as they occur. Methods for optimization of selection on multiple linked QTL developed by Chakraborty et al. [3] and implemented by Dekkers et al. [7] could be used to optimize such selection.

\subsection{Separate male and female lines versus single line selection}

Simultaneous optimal selection within two lines led to greater responses than optimal selection within a single line (Tabs. II and III). Benefits of FULLOPT over SLOPT were less than 2\% for additive QTL but up to $14 \%$ with overdominance. The main reason for these differences is that single line selection limits increases in the frequency of heterozygotes (Fig. 4); although sires and dams can be differentially selected for the QTL within a single line, their joint contributions determine the frequency of the QTL in next generation. As a result, QTL frequencies diverged little between sires and dams with SLOPT, except for the final generations (Fig. 4).

Although optimal selection in two lines resulted in greater responses than optimal selection within a single line, the magnitude of these differences may not justify the extra expenses of maintaining two instead of one line. There are, however, other reasons for having separate male and female lines, including dominance for genes other than those that have been identified, complementarity, and marketing. Even when all QTL that contribute to heterosis have been identified and the majority of the benefit of heterosis can be obtained by optimal selection within a line, especially with limited overdominance, there would still be merit to maintaining separate male and female lines to capitalize on complementarity. Thus, the results presented here provide insight into the 
benefit of and optimal strategies for the use of identified QTL in systems where crossbreeding is the predetermined breeding system.

\subsection{Selective mating on QTL genotype}

In this study, parents for the multiplier and commercial level were randomly selected and mated. Random mating allowed genotype frequencies at the commercial level to be determined entirely by allele frequencies in the nucleus populations, which simplified the model. Selection of multiplier and commercial parents would reduce the polygenic lag between the nucleus and the commercial population. It could also increase the benefit of optimal selection strategies because some changes in QTL frequencies could be accomplished by selection of multiplier parents, which would increase the emphasis placed on polygenic response at the nucleus level. Note that selection of multiplier parents based on the QTL would not require extra genotyping. Additional selection on the QTL among commercial parents would require additional genotyping, although selection at this level could be based on probabilities of QTL genotypes, as determined by genotypes of their nucleus-level parents.

The use of QTL genotype information could be further enhanced by arranging specific matings based on QTL genotype. For QTL with complete and over-dominance, genotyping at the multiplier level would allow mating of opposite homozygotes to maximize the frequency of heterozygotes at the commercial level. This, however, would require extensive genotyping. An alternative is to mate based on probabilities of QTL genotype, as determined by genotypes of nucleus-level parents. Mating at the nucleus level could be arranged to maximize the informativeness of such probabilities. Methods of total genetic resource management described by Kinghorn et al. [11] could be used to develop such strategies.

Note that separate male and female lines would not be required for maximizing the frequency of heterozygotes at the commercial level if mating of commercial parents based on QTL genotype is possible. Instead, much of the advantage of QTL information could be achieved by selection within a single population. Thus, optimal use of QTL information may not require the use of crossbreeding programs, but such programs are often desirable for complementarity and other factor, as discussed previously. In addition, opportunities for selective mating based on observed genotypes or genotype probabilities determined from (grand-)parents are reduced when multiple QTL are selected on simultaneously. 
Li et al. [13] considered a similar population structure as evaluated here but optimized selection and mating of parents for both pure- and cross-breeding. A trait affected by a single gene was considered (no polygenes), which may affect the applicability of their conclusions. Optimization of cumulative discounted response was by differential evolution, which is more flexible in accommodating constraints than the optimal control theory that was used in the present study. Similar to what was observed in the present study, QTL frequencies in the male and female lines diverged for optimal selection, but in a more rapid manner because all emphasis was on the QTL (no polygenes). Li et al. [13] found substantially greater extra cumulative discounted responses in crossbred performance over five generations over a strategy similar to ALTSTD with selective mating than what was observed in the present study: from 5 to $28 \%$ for complete dominance, depending on starting QTL frequencies, and from 11 to $81 \%$ for an over-dominant QTL $(d=2 a)$. For the over-dominant QTL, the benefit was greatest $(81 \%)$ when starting QTL frequencies were equal to 0.5 in both populations. The greater responses can be explained by several factors: not considering polygenes, selection of parents for cross-breeding, and optimization of mating as well as selection. It is unclear to what extent each of these contributed to the extra response. Not considering polygenes results in a large amount of heterosis, especially with overdominance, and does not require simultaneous improvement of polygenes, which increases the benefit that can be obtained from the QTL.

\subsection{Selection on QTL in crossbreeding systems}

Selection on QTL genotype at the nucleus level reduces the need for crossbred testing that is required for combined crossbred and purebred selection (CCPS), thereby saving important test resources and enabling the short generation intervals of purebred selection. Although a two-breed terminal cross was modeled here, results in principle apply to any crossbreeding system.

The results presented here assumed additive polygenic effects, which is unlikely if dominance is observed at identified QTL. Purebred selection based on breeding values derived from phenotype does, however, only act on the additive portion of genetic variance. Thus, presence on non-additive polygenic effects will not change the results of the comparison of QTL versus phenotypic selection when based on purebred data. Non-additive polygenic effects will, however, increase the benefit of using crossbred data in combined crossbred and purebred selection [21]. 
The potential benefit of using crossbred performance for selection in the nucleus through CCPS stems from two sources: non-additive genetic effects and genotype by environment interactions $(\mathrm{G} \times \mathrm{E})$ between the nucleus and the commercial level $[17,18]$. Use of QTL information can capture part and, potentially, all of the benefits from both these factors. The ability to capitalize on non-additive genetic effects with optimized selection on QTL information was demonstrated in the results. QTL information can, however, also address $\mathrm{G} \times \mathrm{E}$ between the nucleus and commercial environments if QTL effects are estimated at the crossbred commercial level, rather than at the nucleus level. Although this would require the availability of data and genotypes at the commercial level, estimates of QTL effects could be obtained from a non-pedigreed sample and would not require routine collection of phenotypes on pedigreed individuals. Thus, the choice between purebred QTL selection and CCPS will depend on the proportion of non-additive genetic variation contributed by the QTL, the impact of other factors that differentiate purebred from commercial performance $(\mathrm{G} \times \mathrm{E})$, the increase in generation intervals associated with CCSP, and the cost of crossbred testing versus that of implementing marker-assisted selection.

The comparisons with phenotypic selection that were presented here apply to a trait of moderate heritability and that is observed in both sexes prior to selection. It is well known that the benefit of marker-assisted selection is greater for traits for which response to phenotypic selection is limited by low heritability and by the unavailability of data on one sex, on selections candidates, or prior to the time of selection. All these limitations apply to the case of selection for crossbred performance using purebred data, in particular in the presence of strong $\mathrm{G} \times \mathrm{E}$, which reduces the accuracy and, thereby, the effective heritability of EBV for crossbred performance. Thus, the benefit of using QTL information will be substantially greater than presented in Tables II and III for additive and non-additive QTL in the presence of $\mathrm{G} \times \mathrm{E}$, in particular when QTL effects are estimated at the crossbred commercial level. The benefit of all QTL selection strategies will increase relative to selection on pure-bred phenotype alone, as the genetic correlation between pure-bred performance in the nucleus and crossbred performance at the commercial level decreases.

In the presence of $\mathrm{G} \times \mathrm{E}$, purebred phenotype is a poor predictor of crossbred commercial performance and thus $\sigma_{E B V}$ based on purebred phenotype will be small, resulting in a large QTL effects relative to $\sigma_{E B V}$. For example, if the genetic correlation between purebred and crossbred commercial performance is $0.5, \sigma_{E B V}$ based on purebred own phenotype with heritability 0.3 would be $0.27 \sigma_{\mathrm{pol}}$ and an additive effect of $3.65 \sigma_{E B V}$, as simulated here for the large 
QTL, would correspond to a QTL with effects on the polygenic and phenotypic scales of $1 \sigma_{p o l}$ or 0.55 phenotypic standard deviations. Thus, even the large QTL simulated here represents a QTL of reasonable effects when put in the context of EBV for crossbred performance, in particular when viewed as the sum of multiple QTL.

\section{ACKNOWLEDGEMENTS}

Financial support from the Pig Improvement Company/SYGEN Int. plc., the NRI Competitive Grants Program/USDA (award No. 98-35205-6736), and the Iowa Agriculture and Home Economics Experiment Station, Ames, Iowa, USA (Project No. 3456), supported by Hatch Act and State of Iowa Funds, is greatly appreciated.

\section{REFERENCES}

[1] Bijma P., van Arendonk J.A.M., Maximizing genetic gain for the sire line of a crossbreeding scheme utilizing both purebred and crossbred information, Anim. Sci. 66 (1998) 529-542.

[2] Bulmer M.G., The Mathematical Theory of Quantitative Genetics, Clarendon Press, Oxford, 1980.

[3] Chakraborty R., Moreau L., Dekkers J.C.M., A method to optimize selection on multiple identified quantitative trait loci, Genet. Sel. Evol. 34 (2002) 145-170.

[4] Dekkers J.C.M., Breeding values for identified quantitative trait loci under selection, Genet. Sel. Evol. 31 (1999) 421-436.

[5] Dekkers J.C.M., van Arendonk J.A.M., Optimizing selection for quantitative traits with information on an identified locus in outbred populations, Genet. Res. 71 (1998) 257-275.

[6] Dekkers J.C.M., Chakraborty R., Potential gain from optimizing multigeneration selection on an identified quantitative trait locus, J. Anim. Sci. 79 (2001) 2975-2990.

[7] Dekkers J.C.M., Chakraborty R., Moreau L., Optimal selection on two quantitative trait loci with linkage, Genet. Sel. Evol. 34 (2001) 171-192.

[8] De Koning D.J., Rattink A.P., Harlizius B., Groenen M.A.M., Brascamp E.W., van Arendonk J.A.M., Detection and characterization of quantitative trait loci for growth and reproduction traits in pigs, Livest. Prod. Sci. 72 (2001) 185-198.

[9] Falconer D.S., Mackay T.F.C., Introduction to Quantitative Genetics, Longman, Harlow, UK, 1996.

[10] Gibson J.P., Short-term gain at the expense of long-term response with selection of identified loci, in: Proc. 5th World Cong. Genet. Appl. Livest. Prod., University of Guelph, Guelph, 21 (1994) pp. 201-204. 
[11] Kinghorn B.P., Meszaros S.A., Vagg R.D., Dynamical tactical decision systems for animal breeding, in: Proc. 7th World Cong. Genet. Appl. Livest. Prod., Montpellier, France, 2002, Communication No. 23-07.

[12] Larzul C., Manfredi E., Elsen J.M., Potential gain from including major gene information in breeding value estimation, Genet. Sel. Evol. 29 (1997) 161-184.

[13] Li Y., van der Werf J.H.J., Kinghorn B.P., Mate selection in parental lines to exploit known dominant QTL in crosses, in: Proc. 7th World Cong. Genet. Appl. Livest. Prod., Montpellier, France, 2002, Communication No. 23-09.

[14] Lynch M., Walsh B., Genetics and analysis of quantitative traits, Sinauer, Sunderland, MA, USA, 1998.

[15] Malek M., Dekkers J.C.M., Lee H.K., Baas T.J., Rothschild M.F., A molecular genome scan analysis to identify chromosomal regions influencing economic traits in the pig. I. Growth and body composition, Mamm. Genome 12 (2001) 637-645.

[16] Manfredi E., Barbieri M., Fournet F., Elsen J.M., A dynamic deterministic model to evaluate breeding strategies under mixed inheritance, Genet. Sel. Evol. 30 (1998) 127-148.

[17] Merks J.W.M., de Vries A.W., New sources of information in pig breeding, in: Proc. 7th World Cong. Genet. Appl. Livest. Prod., Montpellier, France, 2002, Communication No. 03-01.

[18] Merks J.W.M., Hanenberg E.H.A.T., Optimal selection strategy for crossbred performance in commercial pig breeding programmes, in: Proc. 6th World Cong. Genet. Appl. Livest. Prod., Armidale, Australia, 23 (1998) pp. 575-579.

[19] Pong-Wong R., Woolliams J.A., Response to mass selection when an identified major gene is segregating, Genet. Sel. Evol. 30 (1998) 313-337.

[20] Uimari P., Gibson J.P., Combined crossbred and purebred selection vs. pureline selection for a sex limited trait in poultry, in: Proc. 6th World Cong. Genet. Appl. Livest. Prod., Armidale, Australia, 25 (1998) pp. 455-458.

[21] Wei M., van der Steen H.A.M., Comparison of reciprocal recurrent selection with pure-line selection systems in animal breeding (a review), Anim. Breed. Abstr. 59 (1991) 281-298.

[22] Wei M., van der Werf J.H.J., Maximizing genetic response in crossbreds using both purebred and crossbred information, Anim. Prod. 59 (1994) 401-413. 